\title{
Identifying the objects in the structure of an e-model by means of identified formal parameters in the design and engineering environment
}

\author{
Mariya Lavrentyieva ${ }^{l}$, and Aleksey Govorkov ${ }^{1, *}$ \\ ${ }^{1}$ Irkutsk National Research Technical University, 664074, Irkutsk, the Russian Federation
}

\begin{abstract}
This research concerns the issues of automating the design and engineering in aircraft-making. It proposes a method for identifying the structural objects in the e-model of a product by means of formalized parameters. The objects of a part and their interlinkages are described in the form of a structural-geometric model. We therefore analyse the procedure of using this model, whereby production rules as well as set theory are applied. We cite a few basic rules of how to identify the links between individual parts, which may be used to assess the manufacturability of such design, to develop the manufacturing and assembly routes, as well as to design the tooling.
\end{abstract}

\section{Introduction}

The diversity of technical literature and the recommendations featured in the mechanical engineering design analysis [2] contain the basic information about the structure, the composition, and the characteristics of individual parameters used in the analysis. As of today, when designing a product in $\mathrm{CAD} / \mathrm{CAM} / \mathrm{CAE} / \mathrm{PDM}$ systems, it is optimal to analyze the structure of a product in early production, i.e. during design and engineering of aircraft. Thus, to reduce the time it takes to design the documentation and start making the product, the designer and the technologist are tasked to:

- $\quad$ choose modern designs and an optimal option for manufacturing and layout;

- optimally choose the design of the product depending on its intended function;

- use standard, library-provided structural components when modeling a product, say, in NX.

There are no methods and tools for the formal analysis of design composition and structure as such, which prevents automated solution of design and engineering problems. This increases the likelihood of suboptimal solutions, makes the production more time-consuming and labor-intensive.

To solve various problems related to automating the engineering stage of pre-production, we have to design a structural-geometric model of the product.

By using contour recognition method, we can select the geometric primitives that limit the surfaces forming the contour of each structural component

To make recognition more reliable, we used mathematical logic to develop a set of rules that classify the structural components of a part, to find their spatial orientation, their surfaces that face the aerodynamic outlines, their dimensions, etc. In early stages of working with a part, more rules have to be generated because linkages are harder to identify. In terms of design and geometry, a part is uniform and indivisible, its components being non-existent individually; however, in terms of technology, each structural component is individual and independently formable.

The data set obtained enables an engineer to analyze the part as a whole and to identify the nature of its linkages to other parts within an assembly unit, i.e. to find the pairing surfaces and select boundary linkages by means of production rules.

First-level rules are generated in stages [2].

\section{First level rules}

\subsection{Assigning the code depending on the type of lines}

There are straight lines, curves, circles, and arcs. To create a structural combination, we assign each type of those lines a code, see Fig. 1., where: $a$ stands for an arc; $b$ stands for a line; $c$ stands for a circle; $d$ stands for a curve.

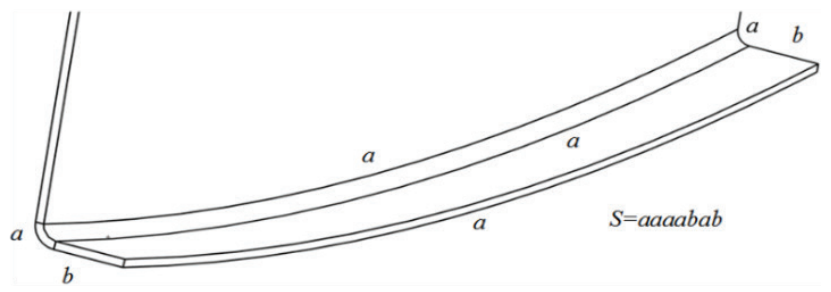

Fig. 1. Components of the code of a board as a structural component

\footnotetext{
* Corresponding author: govorkov as@istu.edu
} 


\subsection{Find the boundary and pairing (crossing) points of the lines.}

Find points gi that are common for various lines $l i$ (at least two points, start point gis and end point gic); find for which structural components ai those points coincide. Each point has coordinates $x, y, z$, i.e. where the coordinates of points belonging to one surface boundary line overlap the coordinates of the points of the boundary line of another surfaces, those surfaces intersect:

$$
\begin{aligned}
& \text { gis }=\text { gjs } \rightarrow\{x i s, y i s, z i s\}=\{x j s, y j s, z j s\} ; \\
& \text { gic }=\text { gjc } \rightarrow\{x i c, y i c, z i c\}=\{x j c, y j c, z j c\} ;
\end{aligned}
$$

where gis and gjs are the starting points of the lines; gic and $g j c$ are the end points.

$$
\text { gis, gic } \in l i \text {; gjs, gjc } \in l j \text {; }
$$

where $l i$ and $l j$ are lines;

$$
l j \in p i ; l j \in p j ;
$$

where $p i$ and $p j$ are surfaces;

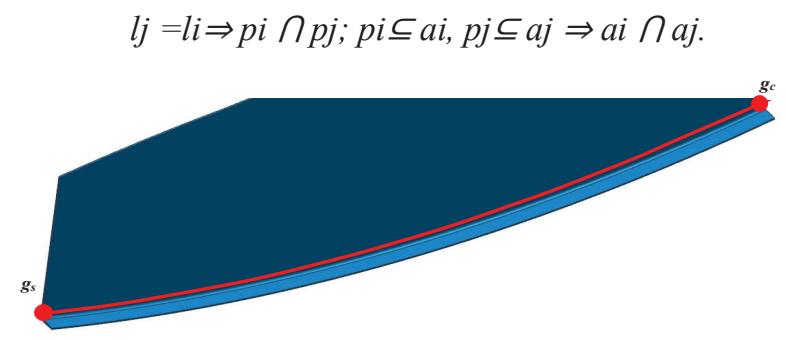

Fig. 2. A common line with start and end points

\subsection{Identify the necessary projections tp}

To identify some structural components unambiguously, we have to analyze its contour in various projections, i.e. for some products, the set of lines may actually consist of multiple sets. This means that if upon the retrieval of primitives, the code of a component coincides with the code of another component while the structural characteristics do not, we need to use additional sets of primitives taken from other projects.

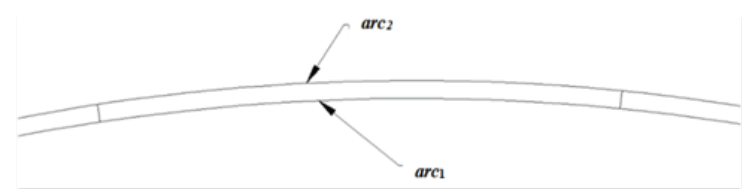

a)

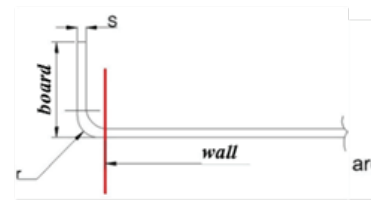

б)

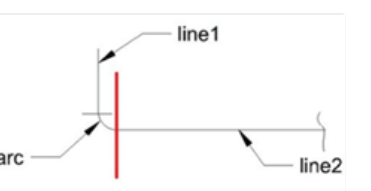

в)

a) longitudinal surface boundary contour of a board; b) transverse surface boundary contour of a board; c) elementary contour dissected into the constituent line types (thickness not factored in).

\subsection{Find the dimensions of lines tp.}

Depending on the type of lines, we find the numeric parameters: length 1 , diameter $D$, radius $R$, and the number of spline segments. Each component contains a set of points, and as mentioned above, each point has its own coordinates, i.e. the length is measurable as a distance between the coordinates of its end points:

$$
\begin{gathered}
g_{i s}=\left\{\begin{array}{l}
x_{i s} \\
y_{i s} \\
z_{i s}
\end{array}\right\}, g_{i c}=\left\{\begin{array}{l}
x_{i c} \\
y_{i c} \\
z_{i c}
\end{array}\right\} ; \\
d t_{\mathrm{p}}=\sqrt{(d x)^{2}+(d y)^{2}+(d z)^{2}},
\end{gathered}
$$

where gis is the starting point of the line; gic is the end point; $t p$ is the inter-point distance.

Transform this expression by multiplying it and dividing it by $d t$ :

$$
d t_{\mathrm{p}}=\sqrt{\frac{(d x)^{2}+\left(d y^{2}\right)+(d z)^{2}}{\left(d t^{2}\right)}} d t
$$

Then divide each term in the numerator by the denominator and present the result as

$$
\begin{gathered}
d t_{\mathrm{p}}=\sqrt{\left(\frac{d x}{d t}\right)^{2}+\left(\frac{d y}{d t}\right)^{2}+\left(\frac{d z}{d t}\right)^{2}} d t \Rightarrow \\
d t_{\mathrm{p}}=\sqrt{\left(x^{\prime}\right)^{2}+\left(y^{\prime}\right)^{2}+\left(z^{\prime}\right)^{2}} d t
\end{gathered}
$$

where $\underline{x}^{\prime}, y^{\prime}$ and $z^{\prime}$ are the derivatives of functions $x(g)$, $y(g)$ and $z(g)$ with variable $g$.

Then

$$
t_{\mathrm{p}}=\int_{g_{i s}}^{g_{i c}} \sqrt{\left(x^{\prime}\right)^{2}+\left(y^{\prime}\right)^{2}+\left(z^{\prime}\right)^{2}} d t .
$$

\subsection{Determine the basic structural component}

The basic structural component $a_{0}$ is the reference point for locating the structural components $a_{0}$ and is characterized by superior overall dimensions $S$, the maximum number of linkages $t_{c}$ with other structural components, the lack of linkages to hanging vertices; it is also the platform for other structural components in the part:

$$
S_{0}>S_{i}
$$

where is $S O$ the area of the basic structural component; $S_{i}$ is the area of a structural component,

$$
t_{c}\left(a_{0}\right)>t_{c}\left(a_{i}\right)
$$

where $t_{c}\left(a_{0}\right)$ is the number of linkages of the basic structural component; $t_{c}\left(a_{i}\right)$ is the number of linkages of a structural component.

Fig. 3. Set of board parameters 




Fig. 4. Determining the basic structural component

\subsection{Orientation of structural components relative to the basic one}

As we have found the basic structural component $a_{0}$, the orientation of other structural components $a_{i}$ shall be determined relative to the basic one made for the zero point of reference.

$$
\begin{aligned}
A_{i} & =\left\{x_{1}, x_{2}, \ldots, x_{j}\right\} ; \\
X_{i} & =\left\{g_{1}, g_{2}, \ldots, g_{i}\right\} ; \\
& G_{i}=\{x, y, z\} .
\end{aligned}
$$

The displacement vector $\bar{k}_{l}$ of the structural component ai relative to $\mathrm{a} 0$ is calculable using the formula:

$$
\bar{k}_{\iota}=\left\{g_{0}-g_{i}\right\}=\left\{\begin{array}{l}
x_{0}-x_{i} \\
y_{0}-y_{i} \\
z_{0}-x_{i}
\end{array}\right\},
$$

where $g_{0}$ is a point of the basic structural component; $g_{i}$ is a point of the structural component to compare.

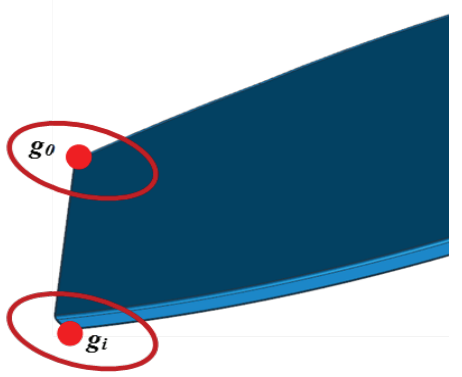

Fig. 5. Points of the base and compared features

\subsection{Facing the aerodynamic outline}

The outline is the surface of the airframe that is streamlines by airflow in flight. The theoretical outline is one that is specified in the theoretical drawings or the mathematical model of the surface. For convenience, denote the outline as $w$. The outline surface is formed by the surfaces of the structural components belonging to the parts that form the actual outline as a result of assembly.

Whether this or that structural component $a_{i}$ belongs to the outline $\mathrm{w}$ in the theoretical e-model (TEM) of the product can be found out using the formula:

$$
a_{i} \epsilon w_{i} f p\left(a_{i}\right) \cap p(w) .
$$

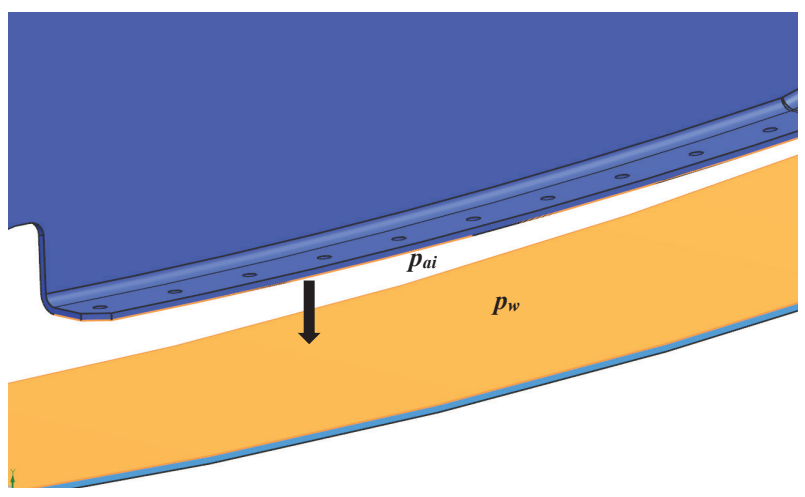

Fig. 6. Outline surface and board surface, paired

As mentioned above, we use the contour recognition method that underlies this set of rules. These rules are a minimum for generating the second-level rules because it enables us to identify the types of the structural components of a part, their location in relation to each other, by linking the primitives, the number of components of the same type, and the total number of the structural components in a part.

For a broader picture of the structure of a part, its structural components are classified to optimize the product of modeling by reference to the identified specifics of construction [1].

Thus, the identified design and engineering parameters match a specific design component, the curvilinear board in our case. This identification fits each and every structural component of the part, which further enables the classification of the part itself.

Part classifier is designed to select a specific group or family based on whether the part has or lacks specific properties.

Considering the second-level operations with the product, recognition as such is not used in this set of rules because the use of the solution to the first problem combined with production rules enables the analysis of parts in the assembly context. In this case, we only need to formulate a few basic rules to identify the linkages between individual parts. 10].

Second-level rules consist of the following stages [1,

\section{Second-level rules}

\subsection{Determining the basic part}

Which part shall be the basic part (a rigid part or a lowrigidity part) determines to a great extent the sequence and the method of assembly; this is the basis, upon which the basic component identification criteria are determined.

As with the basic structural component, the basic part has the largest number of linkages $t_{c}$ and as a rule, has superior overall dimensions $S_{b e}$.

$$
S_{b e}>S_{e}
$$

where $S_{63}$ is the area of the basic component;

$S_{\ni}$ is the area of a component, 


$$
t_{c}\left(m_{0}\right)>t_{c}\left(m_{i}\right)
$$

where $t_{c}\left(m_{0}\right)$ is the number of linkages of the basic part; $t_{c}\left(m_{i}\right)$ is the number of linkages of a part.

\subsection{Finding all the pairing surfaces of various components}

We have to find all the pairing surfaces for each component to make the final decision regarding the type of a structural component, which has been identified when solving the first-level problem. To find out whether the surfaces are paired, at least three points of each surface should share their coordinates.

$$
\begin{aligned}
& g_{i 1}=g_{j 1} \rightarrow\left\{x_{i s}, y_{i s}, z_{i s}\right\}=\left\{x_{j s}, y_{j s}, z_{j s}\right\} \\
& g_{i 2}=g_{j 2} \rightarrow\left\{x_{i c}, y_{i c}, z_{i c}\right\}=\left\{x_{j c}, y_{j c}, z_{j c}\right\} \\
& g_{i 3}=g_{j 3} \rightarrow\left\{x_{i c}, y_{i c}, z_{i c}\right\}=\left\{x_{j c}, y_{j c}, z_{j c}\right\}
\end{aligned}
$$

where $g_{i s}$ and $g_{j s}$ are the starting points of the lines; $g_{i c}$ and $g_{j c}$ are the end points.

$$
\begin{aligned}
& g_{i 1}, g_{i 2}, g_{i 3} \in p_{i} ; \\
& g_{j 1}, g_{j 2} g_{j 3} \in p_{j} ;
\end{aligned}
$$

where pi and pj are surfaces;

$$
p_{i} \cap p_{j}
$$

\subsection{Identification of components}

After we have finally determined the composition of structural components and the pairings of their surfaces, we can identify the type of part this component belongs to.

$$
m_{i}=m_{1} \rightarrow m_{i}=\left\{a_{1-1}, a_{1-2}, a_{1-i}\right\},
$$

where $m_{i}$ is any part; $m_{1}$ is the part to identify.

\subsection{Spatial orientation of components relative to the basic one}

As all parts have been identified, and their pairing surfaces established, we can find out their coordinate position relative to each other and get an idea of the general contour and dimensions of the product as a whole. Unlike structural components, each part has a single point that can be used to find the spatial position thereof. The point is the center of mass point $g_{u}$, and the position is found using the displacement matrix $M_{p e r}$ relative to the coordinate system used in the assembler file.

$$
M_{\text {per }}=M_{r} \times M_{v}
$$

where $M_{p e r}$ is the displacement matrix; $M_{r}$ is the rotation matrix; $M_{v}$ is the transition matrix.

$$
M_{\text {per }}=\left|\begin{array}{ccc}
x x_{0} & x y_{0} & x z_{0} \\
y x_{0} & y y_{0} & y z_{0} \\
z x_{0} & z y_{0} & z z_{0}
\end{array}\right| \times\left|\begin{array}{c}
x_{v} \\
y_{v} \\
z_{v}
\end{array}\right|,
$$

$$
\left|\begin{array}{l}
x_{v} \\
y_{v} \\
z_{v}
\end{array}\right|=\left|\begin{array}{l}
x_{0}+x_{i} \\
y_{0}+y_{i} \\
z_{0}+x_{i}
\end{array}\right|
$$

Solving the second-level problem helps get an idea of the product in general and of the structure and composition of each of its parts. In terms of methodology, there is no familiar semantic binding to a particular denomination; we merely assign a type of part and therefore obtain a product consisting of $n$ parts of determined composition and structure.

To automate structural component data processing, it makes sense to use all-Russian technological process and transition classifiers.

The sets of components in such parts differ, which means we have to model and manufacture them in different ways.

Determination of the design and engineering parameters of a part as well as classifying it as a certain type have a considerable impact on its subsequent basing. We introduce several rules.

\section{Third-level rules}

\subsection{Determining the composition of structural components ai}

According to study cases, typical parts have certain structural components unique to this type of part:

$$
\begin{aligned}
& a_{i 1}, a_{i 2}, a_{i 3} \in m_{i}, \\
& a_{j 1}, a_{j 2}, a_{j 3} \in m_{j},
\end{aligned}
$$

where $m_{i}$ is an $i^{\text {th }}$ type part; $m_{j}$ is a $j^{\text {th }}$ type part.

\subsection{Determining the semi-finished product}

When finding out the structural and engineering properties of a part, the choice of the semi-finished product is very important; by default, profile semiproducts are considered more rigid than sheet semiproducts; a wall made from a plate is more rigid than one made from a sheet.

\subsection{Finding the constant thickness $s$ of a part}

Beside the physical and mechanical properties of semifinished products, some of which are added to the e-model when designing it, the thickness of material is of importance, too; as a rule, sheet-made parts have a constant thickness, while plate-made ones may have significant variations in thickness.

\subsection{Finding the center of mass point $g_{u}$}

The center of mass point has its own coordinates in each individual part. It is a reference point for finding the location and pairings of parts in an assembly unit. The center of mass coordinates are the closest for two parts of similar dimensions and a complete pairing on one surface: 


$$
g_{u}=\left\{x_{u}, y_{u}, z_{u}\right\},
$$

where $\left\{x_{u}, y_{u}, z_{u}\right\}$ are the coordinates of the center of mass..

Thus, a classification enables clear grouping of parts, which enables using them to create basic models, analyze their typical formulation, and identify the most optimal option.

As noted above, all parts under analysis that form the structure of nodes and units in an aircraft can be rationally represented as combinations of typical structural components. The choice of their composition, design options, the analysis of their compatibility enable a formalization of the recognition process for each specific part and the entire assembly unit.

We therefore obtain a classified product not bound to semantics, with a complete inventory of parameters selected on the basis of the rules developed [10]. Rules for selecting the relevant parameters are in its turn formulated depending on the ultimate purpose; they enable further transfer of data for the desired output.

The research herein presented is supported financially by the Fund for the Promotion of Small Scientific and Technical Enterprises as a part of the project 'Developing a System for Automated Analysis of Aircraft Engineering Products in Preproduction', the UMNIK 16-10 contest.

\section{References}

1. S.A. Aksenov, E.N. Chumachenko, A.V. Kolesnikov, S.A. Osipov, Determination Of Optimal Conditions For Gas Forming Of Aluminum Sheets, Procedia Engineering 11 th., P. 1017-1022 (2014)

2. S.A. Aksenov, I.Y. Zakhariev, A.V. Kolesnikov, S.A. Osipov, Characterization of superplastic materials by results of free bulging tests, Materials Science Forum. Vol. 838-839, P. 552-556 (2016)

3. P.E. Chimitov, Generating the image of a product for designing the airframe assembly process. Reshetnyov's, Readings: Proceedings of the XII International Scientific Conference in the Memory of the Designer General of Rocket Space Systems, Academician M. F. Reshetnyov, P.183-184 (2008)

4. V.E. Gozbenko, S.K. Kargapoltsev, D.N. Kornilov, N.V. Minaev, A.I. Karlina, Definition of the main coordinates of the car with two-level spring suspension, International Journal of Applied Engineering Research. Vol. 11. Issue 20, P. 1036710373 (2016)

5. V.A. Grechishnikov, R.M. Khusainov, D.R. Akhkiyamov, S.Y. Yurasov, O.I. Yurasova, Identifying the primary rigidity axes in the elastic system of a metal-cutting machine, Russian Engineering Research Vol. 36, No. 8, pp. 673-67 (2016)

6. G.K. Irzaev, Expert methods of industrial products manufacturability management (Moscow: InfraInzheneria, 2010)
7. G.K. Irzaev, Research and modeling data streams of design and engineering adjustments in the development and medium-batch manufacturing of products, Organizator proizvodstva Vol. 52. Issue 1, P. 131-135 (2012)

8. G.K. Irzaev, Expert methods for controlling the manufacturability of industrial products: a monograph (Moscow: Infra-Inzheneriya, 2010)

9. A.A. Kabanov, Object model for the analysis of aircraft products with account of inheritance for the assessment and control of the efficiency of manufacturing systems in discrete mechanical engineering at the stage of their organizational and engineering design or modernization, Kazan: Scientific and Technical Bulletin of the Volga Region, p. 161-168 (2014)

10. R.M. Khusainov, I.F. Sharafutdinov, Methods of assessing the dynamic stability of the cutting process using Unigraphics NX, IOP Conf. Series: Materials Science and Engineering 134, 012042 (2016)

11. P.M. Krastyaninov, R.M. Khusainov, Selection of equipment for machining processing of parts using NX and Teamcenter programs, IOP Conf. Series: Materials Science and Engineering 134, 012041 (2016)

12. A.A. Mitin, Use of production rules to display the integrity limits in graphical CAM editors, Proceedings of the Oryol State Technical University. Series: Information Systems and Technology. Oryol: Oryol State Technical University, P.173-177 (2008)

13. K.A. Odnocurtcev, Laboratory equipment for automated control of assembly tooling components, Bulletin of Irkutsk State Technical University. Issue 12 (59), P. 41-47 (2011).

14. A.K. Shmakov, V.V. Mironenko, K.K. Kirishina, A. S. Stanislavchik, V.V. Kotov, Effect of the average velocity of the free part of the semifinished product on the process of pneumothermal forming in the superplastic regime, Metallurgist. -Vol. 57, Issue 12, P. 8-12 (2013) 